\begin{tabular}{c|c|c|c|c|c|c|c|} 
DOI: http://dx.doi.org/10.21276/ap.covid19.2021.10.1.15 \\
Annals of Phytomedicine: An International Journal \\
http://www.ukaazpublications.com/publications/index.php \\
Print ISSN : 2278-9839
\end{tabular}

\title{
Current understanding of gut microbiota in tackling COVID-19
}

\author{
S. Sumathi», K. Suganya, K. Swathi, B. Sudha and S. Sneha \\ Department of Biochemistry, Biotechnology and Bioinformatics, Avinashilingam Institute for Home Science and Higher Education for \\ Women, Coimbatore-641043, Tamil Nadu, India
}

\section{Article Info}

\section{Article history}

Received 7 March 2021

Revised 27 April 2021

Accepted 29 April 2021

Published Online 30 June 2021

\section{Keywords}

COVID-19

SARS-CoV-2

ACE2 receptors

Respiratory viruses

Pathogenesis

Gut microbiome

Immunity

Diet

\begin{abstract}
SARS-CoV-2 is a global pandemic that mainly affects the respiratory system by binding to host cell transmembrane protease serine-2 (TMPRSS-2) and ACE2 (angiotensin-converting enzyme) receptors on alveolar epithelial cells. The gut microflora plays an important role in maintaining human health and of greatest concern in covid affected individuals. Dysbiosis may be a consequence for lung infection that has been linked to the severity of SARS-CoV-2. Recent studies identified the presence of SARSCoV-2 RNA in the faeces of COVID-19 patients, which has been associated with several gastrointestinal tract ailments and multiorgan dysfunction. Diet, lifestyle, and genetics plays a vital role in determining the gut microbiota and immune compromise to the virus infection. COVID-19 is attributed to the variations in gut microbiota diversity which often decreases with age and, hence the lethality in elderly patients. Intestinal microbiota profile can be enhanced by improved diet and supplementation, which has been shown to increase immunity in elderly and immune-compromised patients. Diet has an incredible impact on the gut microbiome, allowing for a new state of homeostasis to be achieved through intake timing, frequency, and duration. This review article focuses on gut, lung microbiota, and immunomodulation, with an emphasis on gut microbiota-induced immune activation.
\end{abstract}

\section{Introduction}

The gut microbiota is a complex species of commensal bacteria that lives in the human gastrointestinal (GI) tract. The number of microorganisms present in the GI tract is estimated to be greater, with 100 times the amount of genomic content (microbiome) as the human genome. Depending on environmental and genetic factors, the gut microbiome comprises 1000 species of bacteria, with an individual containing approximately 160 species. In the gut, Firmicutes and Bacteroidetes predominate, while in the lungs, Proteobacteria, Bacteroidetes, and Firmicutes predominate (Wang et al., 2020). Gut microbiota has provided numerous benefits to its host over time, including direct pathogen inhibition, gut integrity maintenance, metabolizing undigested substances, particularly certain carbohydrates, and the production of tolerant mucosal barrier and intestinal epithelium. Since the gut contains $70-80 \%$ of the body's immune cells, the immune system and gut microbiota have a complex interplay and relationship that controls and supports each other (Xiao et al., 2020). Dysbiosis, characterized as changes in gut microbiota that result in microbial imbalance, has been linked to the pathogenesis of many inflammatory diseases and also plays a role in a variety of infections. Viruses are one of the most common pathogens that cause strong interactions between viruses and the commensal microbiota (Li et al., 2019).

\footnotetext{
Corresponding author: Dr. S. Sumathi

Associate Professor, Department of Biochemistry, Biotechnology and Bioinformatics Avinashilingam Institute for Home Science and Higher Education for Women, Coimbatore-641043, Tamil Nadu, India E-mail: sumathi_bc@avinuty.ac.in

Tel.: +91-9843021733
}

Copyright $(\odot) 2021$ Ukaaz Publications. All rights reserved.

Email: ukaaz@yahoo.com; Website: www.ukaazpublications.com
The severe global burden of disease caused by COVID-19 infection has prompted almost unparalleled levels of rapid activity across various disciplines by the international scientific community in an effort to understand pathogenesis and establish therapeutic options for this novel virus, with an emphasis on slowing and preventing its transmission (Shen et al., 2020). The marked heterogeneity of clinical presentation in COVID-19 infection is particularly intriguing, with certain infected individuals being asymptomatic while others progress to multiorgan damage and death. A comparison of clinical, molecular, and immunological data from a large Chinese cohort of COVID-19 patients revealed that viral genetic diversity did not even appear to be correlated with disease severity, whereas host factors (such as age and inflammatory response) appeared to play a much prominent role (Kalantar Zadeh et al., 2020).

The gastrointestinal (GI) tract has been implicated in a variety of emerging clinical and scientific strands of research as a significant organ for the susceptibility to, and intensity of COVID-19 infections. In addition to normal respiratory and constitutional symptoms, GI symptoms (such as nausea, vomiting, and diarrhoea) have been consistently identified as common clinical features of infection $(\mathrm{Wu}$ et al., 2019). COVID-19 has also been found in tissues from throughout the GI tract, and virus shedding in stool has been observed in a significant proportion of patients, with shedding often enduring for long periods of time; raised faecal calprotect in has also been reported in association with infection (Cai et al., 2020). Further more, evidence from organoid models suggests that COVID-19 can specifically infect the GI tract; the SARS-CoV-2 virus, for example, uses angiotensin converting enzyme 2 (ACE-2), which is highly expressed on differentiated enterocytes, as a receptor for cell entry before inducing a viral response program (Fang et al., 2020). 
In this study, we have discussed how the gut microbiome plays a role in the pathogenesis of COVID-19 infection and the effects of the SARS-CoV-2 virus's interaction with the gut microbiome.

\section{Dysbiosis and COVID-19}

The human gastrointestinal (GI) tract contains up to 2000 bacterial species divided into 12 phyla, with the Proteobacteria (Gramnegative), Firmicutes (Gram-positive), Actinobacteria (Gram-positive) and Bacteroidetes (Gram-negative), phyla being the most common (>90\%) (Hugon et al., 2015; Li et al., 2014). With the diversity of microbes, a new concept of "gut virome" has emerged, which is need to be considered as a significant aspect of a healthy human microbiota (Scarpellini et al., 2015). The human gut virome almost shares the vital information with all microbial components and may have an effect on overall human health by shaping gut community structure and function (Cani, 2018; Garmaeva et al., 2019). Gut eukaryotic viruses that are most commonly seen during viral gastrointestinal infections include Norwalk, Rotaviruses, Enteroviruses (the wellknown agents of gastroenteritis in humans) and in recent time, SARSCoV-2 infection (Scarpellini et al., 2015).

Dysbiosis, characterised as changes in gut microbiota that result in microbial instability, has been linked to the pathogenesis of many inflammatory diseases and also plays a role in a variety of infections. Dysbiosis of the gut microbiota causes abnormal immune response in the respiratory epithelium and mucosa, which may cause immune responses to be compromised for respiratory viral infections (Musa, 2020). Many studies have documented the gastrointestinal symptoms as one of the major clinical features during the course of the illness (Zhang et al., 2020a), and perhaps even the presence of viral RNA in faeces (Ng and Tilg, 2020; Xiao et al., 2020). SARS-CoV-2 requires the presence of transmembrane protease serine-2 (TMPRSS-2) and angiotensin converting enzyme-2 (ACE-2) receptor in enterocytes from the colon and ileum to enter and infect the cells (Hoffmann et al., 2020; Zhang et al., 2020b). These gastrointestinal changes in COVID-19 may be a consequence of the major pulmonary changes, or SARS-CoV-2 may be engulfing enterocytes, causing gut dysbiosis and increased negativity of lung homeostasis. The gut microbiota of the elderly (in particular those with age-related pathologies, e.g., cardiovascular, metabolic, renal diseases) is normally less oriented (Shen, 2017), which could be related to the more severe effect of SARS-CoV-2 infection among elderly people that share the common phenomena of gut microbiome dysbiosis. As a result, there appears to be a bidirectional relationship between SARS-CoV-2 infection and dysbiosis, not only as a result of SARS-CoV-2 infection, but also as a possible risk factor for Covid-19 negative consequences (KalantarZadeh et al., 2020).

Intestinal gut microbiome is regulated by innate and adaptive immunity through certain mechanisms of antimicrobial peptides and $\operatorname{IgA}$ antibodies. Imbalance in the microbiome alters the innate and adaptive immunity, thereby affecting the colonization of microbiota (Xu et al., 2020). A recent study from China showed that COVID-19 patients had gut dysbiosis with lower levels of Bifidobacterium and Lactobacillus, both are probiotic strains (Kuba et al., 2005). ACE2 controls the expression of amino acid transporter B(0)AT1, which is responsible for the intake of tryptophan (Zhao et al., 2018) and also the antimicrobial peptides' mRNA expression is controlled by tryptophan via the mTOR pathway (Lie'vin-Le Moal and Servin, 2006) which can impact the composition of the gut microbiota.
During infection, ACE2 receptor expression becomes downregulated, thereby as a consequence, normal absorption of tryptophan by intestine is reduced and also affects the normal mechanism of antimicrobial peptides, resulting in greater pathogen sustenance and gut dysbiosis (Zuo et al., 2020a). Figure 1 shows the influence of gut microbiota in COVID-19 immune homeostasis.

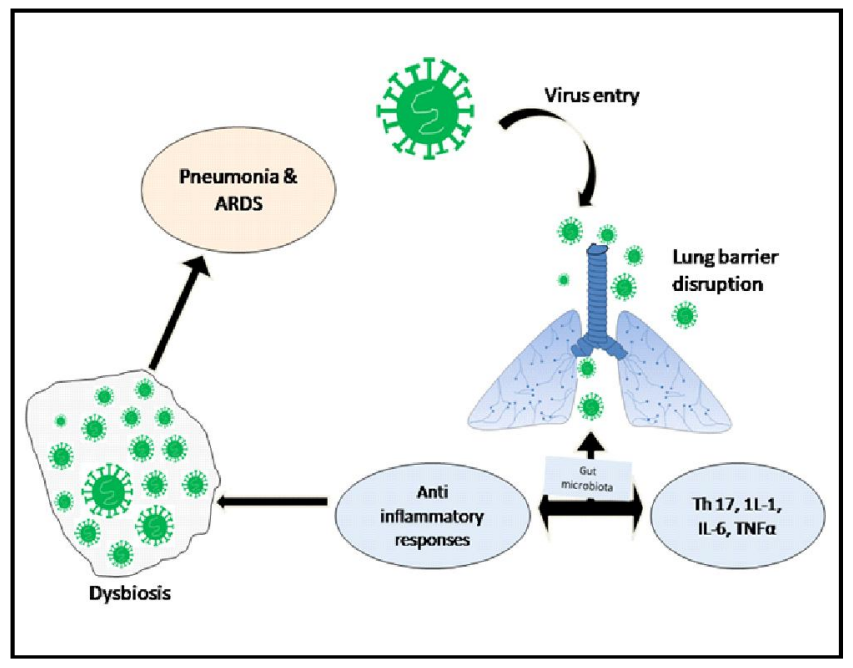

Figure 1: Influence of gut microbiota in COVID-19 immune homeostasis.

A shotgun metagenomic analysis of COVID infected patients' faecal samples reported a decrease in beneficial microflora and an increase in pathogenic strains. This gut dysbiosis was observed even after the clearance of respiratory illness. Clostridium hathewayi and Clostridium ramosum are responsible for bacteremia and clinical severity (which includes pneumonia along with fever and respiratory tract symptoms; respiratory ratee $\geq 30 / \mathrm{min}$, oxygen saturationd $\geq$ 93\%) and the genus Coprobacillus has been shown to strongly upregulate colonic ACE-2 receptor (Yang et al., 2020). Due to the severity of infection by SARS-CoV-2, the Faecalibacterium prausnitzii, Alistipes onderdonkii, Roseburia and Lachnospiraceae, which are involved in maintaining gut and inflammatory homeostasis might decline, leading to dysbiosis. A study conducted in SARS-CoV-2 individuals showed that Bacteroides thetaiotaomicron, Bacteroides dorei, Bacteroides ovatus and Bacteroides massiliensis were found to have a strong negative association with faecal viral load (Elsayed and Zhang, 2004) and they are all attributed to a decrease in ACE-2 expression in the colon (Yang et al., 2020). There was a higher rate of increase in pathogenic organisms including Actinomyces, Rothia, Sterptococcus, and Veillonllea as well as a decrease in beneficial microflora in covid infected patients was reported (Gu et al., 2020). Multiple bacterial flora that are beneficial to host immunity, such as Faecalibacterium prausnitzii, Lachnospiraceae bacterium, Eubacterium rectale, Ruminococcus obeum and Dorea formicigenerans showed a further decline in SARS-CoV-2 infected people (Zuo et al., 2020a).

A study from Wuhan, China studied the association between dysbiosis and covid infection severity (Gou et al., 2020). Elevated levels of proinflammatory bacterial strains, such as Klebsiella, Streptococcus and Ruminococcus gnavus were associated with the higher levels of proinflammatory cytokines and expanded disease 
severity. These microbes had previously been discovered to be enriched in the proinflammatory intestinal tract of patients with diabetes, obesity, irritable bowel disease (IBD), and high blood pressure. Most of the inflammatory cytokines studied have a clear positive association with Ruminococcus gnavus. $R$. gnavus, in particular, produces a proinflammatory glucorhamnan polysaccharide that powerfully induces TNF (tumor necrosis factor) secretion by dendritic cells via Toll-like receptor 4 (Henke et al., 2019). R. gnavus can also destroy mucin to be used as a carbon source (Png et al., 2010; Bell et al., 2019), causing the disintegration of mucus layer and its intestinal barrier function. A dysbiotic gut microbiome with high levels of Streptococcus and lower incidence of Lactobacillus species was identified in Kawasaki disease (Esposito et al., 2019), a disorder similar to multisystem inflammatory syndrome in children (MIS-C) that is increasingly identified as a complication in young children diagnosed with COVID-19 (Jones et al., 2020). As a result, changes in the gut microbiome caused by COVID-19 may play a role in this complication

Han et al. (2020) looked into whether the SARS-CoV-2 infection has an effect on lung microflora, which could lead to COVID-19 complications. The findings suggest that SARS-CoV-2 infection has a significant impact on the lung microbiota. COVID-19 subjects had severe microbiota dysbiosis, with elevated levels of pathogenic bacteria like Klebsiella oxytoca, Lactic acid Bacteria, Faecalibacterium prausnitzii and Tobacco Mosaic Virus (TMV). In the lungs, a harmful inflammatory atmosphere was discovered, which was linked to Rothia mucilaginosa and SARS-CoV-2. This preliminary evidence suggests that the lung microbiota plays a role in the SARS-CoV-2 infection process and may help researchers better understand the virus prevention and complications.

A mice model study proved the modulation of gut microbiota or an induction of dysbiosis by ACE2 receptor from colon (Yang et al., 2020). Fusicatenibacter, Romboutsia, Intestinibacter, Actinomyces and Erysipelato clostridium were also found to be a differentiating factor of symbionts or a biomarker between the COVID-19 community and the healthy individuals. A shotgun metagenomics analysis with baseline stool samples from COVID-19 patients revealed a reduction in Faecalibacterium prausnitzii species and altered host immune response (Geva-Zatorsky et al., 2017). Erysipelotrichaceae bacterium, responsible for GI tract inflammation was found to be a strong hausser of SARS-CoV-2 infection in the host gut. Another metagenomic analysis of faecal samples from virus infected patients showed a significant variation in their microflora enriched with Candida albicans, Candida auris, Aspergillus flavus and a heterogenous mycobiota (Zuo et al.,2020a). Viral RNA metagenomic sequencing of faecal samples from SARS-CoV-2 patients showed the active viral load for 6 days even after the clearance of nasopharyngeal SARS-CoV-2 with high incidence of Collinsella aerofaciens, Collinsella tanakaei, Streptococcus infantis and Morganella morganii (Zuo et al., 2020b). Another study suggested the impact of gut bacteria on colonic ACE2 expression and systemic inflammation in their hosts. Yang et al. (2020) found a reduction in colonic ACE2 expression, when gut microbiota is re-established in germ-free rats. Higher levels of tryptophan metabolites, kynurenic acid, and hydroxykynurenine, were discovered in serum metabolome research, implying that these metabolites are involved in ACE2 control. This suggested that the gut microbiota may influence intestinal ACE2 expression and contribute to dysbiosis in SARSCOV-2 infected host (Yang et al., 2020).

\section{Respiratory viruses and gut microbiome}

Bradley et al. (2019) recently found that influenza virus highly influence the gut microbiota which is highly responsible for the viral infection of respiratory tract. Type 1 IFN-1 is target driven signal of gut microbiome and it fights against premature viral infections (Steed et al., 2017). Influenza virus multiplies in the respiratory tract of COVID-19 patients, this viral infection was considered as a clinical spectrum (Pan et al., 2020). Viral infection has changed the composition of gut microbiome with the help of type 1 IFN-I molecules. Remarkably, IFN-I molecules induced by influenza virus supports the reduction of anaerobic bacteria and improve the Entero bacteriaceae in the gut which inactivate viral mechanism (Pan et al., 2016).

Proinflammatory gut microbiome modification has happened because of the influence of respiratory viruses like adenovirus. In adenovirus infection in lemur model, the count of essential healthy gut microbiome was very low whereas the count of genera contain pathogen like Nessieria genera was high (Wasimuddin et al., 2019).

In these cases, gastrointestinal symptoms where seen to show the severity of COVID-19 infection (Li et al., 2019). Dysbiotic gut microbial condition and epithelial swelling boosts the angiotensinconverting enzyme 2 (ACE2) level, which is the cell surface receptor molecule involved in the dietary amino acid homeostasis, innate immunity, and gut microbial ecology (Hashimoto et al., 2012). Target of SARS-CoV-2 was ACE2 (Chan et al., 2020), elevated level of ACE2 from patients were characterized by proinflammatory gut microbiome highly supports the coronavirus infections like SARS-CoV (Wang et al., 2020). In that, SARS-CoV could spread throughout the human body (Zhou et al., 2017). Researchers have identified viral RNA from the feces of many COVID-19 positive patients. With this, they identified development of infection in the gastrointesinal tract (Xiao et al., 2020).

Digestive symptoms and respiratory symptoms of COVID-19 patients were compared for the confirmation and presence of viral load in the fecal sample (Han et al., 2020). Extended digestive clinical spectrum like diarrhea is not connected with the composition and richness of gut microbiota. So, the modification of gut microbiota is directly related to immune dysfunction (Vandeputte et al., 2016). This finding supports the redesigning the gut microbiota would be adjuvant therapy for those who are suffering from digestive symptoms associated with COVID-19.

The connection between patients's with unbalanced microbial composition and healthy individual microbial population was confirmed (Gou et al., 2020). Higher level of Lactobacilllus species associated to increased proportion of anti-inflammatory interleukin10 enhanced the disease prediction. High level of proinflammatory bacterial species like Streptococcus, Klebsiella and Ruminococcus gnavus correlated with the proinflammatory cytokines and enhanced the disease seriousness. The above mentioned organisms were enriched in gut microbiota of those who are suffering from diabetes, obesity, irritable bowel syndrome and high blood pressure.

Kawasaki disease is a one of the complication in children associated with COVID-19 (Jones et al., 2020), is characterized by a dysbiotic gut environment including high count of Streptococcus and lesscount of Lactobacillus compared to healthy children (Esposito et al., 2019). These studies show that COVID-19 stimulates the alteration in composition of gut microbiome mayroute to this barrier. 


\section{Disruption of the gut microbiome and COVID-19}

SARS-CoV-2 enters the host via the angiotensin converting enzyme 2 (ACE2) receptor, which is strongly expressed in the respiratory and gastrointestinal tracts. Intestinal inflammation and gut microbial environment, both are regulated by ACE2 (Hill et al., 2020). The gut microbiome, which contains trillions of different bacteria, has a wide range of impacts on gene regulation, immune response, and metabolism. Invading viruses can manipulate the commensal microbiota environment in the gut to facilitate a stimulatory or suppressive response. Respiratory virus infections have been linked to changes in the gut microbiome, which may predispose patients to secondary bacterial infections, according to research (Mendes et al., 2019). Pathogens or oral and upper respiratory commensal bacteria dominated the microbiota in SARS-CoV-2 infected patients, according to recent meta-transcriptome sequencing of bronchoalveolar lavage fluid. Furthermore, changes in bacteria taxa from the phyla Bacteroidetes and Firmicutes, which have been shown to control ACE2 expression in rodents, have been linked to co-morbidities usually associated with extreme COVID-19. Understanding the host microbial disturbances that cause SARS-CoV-2 infection is critical because it could affect infection response and the effectiveness of potential immune interventions such as vaccines (Hagan et al., 2019).

Loss of beneficial species in COVID-19 persisted in the number of people despite SARS-CoV-2 virus clearance, indicating that SARSCoV-2 infectionand/or hospitalization could be linked to a longerterm negative impact on the gut microbiome. Respiratory viral infections, such as influenza and respiratory syncytial virus (RSV), have been shown in studies to modify the gut microbiome (Zimmermann et al., 2019). Patients with viral infections are more likely to develop secondary bacterial infections, which have a more serious clinical path. Clostridium hathewayi, Bacteroides nordii, and Actinomyces viscosus was the opportunistic pathogens found in the gut microbiome of COVID-19 patients, and a higher baseline abundance of Clostridium hathewayi was linked to more extreme COVID-19. The majority of these bacteria are bacteraemia-associated bacteria, implying a risk of a serious disease path due to secondary viral infection (Matson et al., 2018). Actinomyces viscosus, an opportunistic pathogen of the oral cavity and upper respiratory tract, has been found in the guts of COVID-19 patients. Its existence indicates that extraintestinal microbes have passed through or been transmitted through the gut (Wei et al., 2018).

SARS-CoV-2 can bind to human ACE2 as a host entry point, according to a study published recently. ACE2 regulates amino acid transport, microbial ecology, and inflammation in the gut and is highly expressed in the intestine, especially in colonocytes of healthy subjects and patients with inflammatory bowel disease. Bacteroidetes species have been shown to suppress ACE2 expression in the murine colon, while Firmicutes species modulate ACE2 expression in a variety of ways (Doan et al., 2019). Bacteroidetes species Alistipes onderdonkii and Bacteroides ovatus had a negative association with COVID-19 intensity, and four species from the phylum Bacteroidetes (Bacteroides dorei, Bacteroides thetaiotaomicron, Bacteroides massiliensis, and Bacteroides ovatus) had an inverse correlation with SARS-CoV-2 fecal viral load. Bacteroides dorei is one of them, and it has been shown to suppress colonic ACE2 expression and calibrate the host immune response. Older patients and those with underlying chronic conditions associated with inflammation, such as hypertension, obesity, diabetes mellitus, and coronary artery disease, had the highest SARS-CoV-2 mortality and morbidity. Surprisingly, these individuals were found to have a lower abundance of Bacteroides species than healthy people. All of these results lead to the fact that a person's gut microbiome configuration influences their vulnerability and response to SARS-CoV-2 infections (Strathdee et al., 2020).

Increased interferon-inducible protein and other cytokines were included in the cytokine profile correlated with hyperinflammation in extreme COVID-19. Understanding host cytokine pathways and microbiota interactions with cytokine reactions in SARS-CoV-2 infection is critical in developing new therapeutic methods, given the lack of proven treatments for COVID-19 (Bradley et al., 2019). The use of empirical antibiotics (which was common during the initial SARS-CoV-2 outbreak when secondary bacterial infection was a challenge) resulted in further loss of beneficial symbionts and exacerbation of gut dysbiosis in COVID-19 patients, and our findings support the avoidance of unnecessary antibiotics in the treatment of viral pneumonitis, as antibiotics can eliminate beneficial bacteria and weaken the immune system. In addition, antibiotic-induced changes in the gut microbiome can affect human vaccine immunity. In order to combat COVID-19, it is necessary to consider improving the effectiveness of potential immune treatments such as vaccines by modulating the gut microbiome (Baud et al., 2020). One approach for promoting a healthy microbiome may include measures to enhance intestinal butyrate production through the promotion of microbial interactions by dietary changes, and reduction of pro-inflammatory states. These data highlight a new concept that novel and targeted approach of modulation of the gut microbiota may represent a therapeutic avenue for COVID-19 and its co-morbidities (McIlroy et al., 2020).

\section{Microbiome as a barrier to virus infection}

The micro-organism population has greatly evolved the human species over many millions of years. Because of the bacterial coevolution, eukaryotic cells could produce genomic functional complementarity with genetic disease (Zilber-Rosenberg and Rosenberg, 2008). Cells and microbes interact in environments that are based on fluctuating competitive and collaborative patterns in and between species. Cells receive and transmit chemical messages that modify expression of genes and cellular activities, transmit biomolecules and metabolites that strengthen the community's persistence and feasibility, and trigger defense mechanisms to regulate species contending for same important resources in order to sustain these balances (Caruana and Walper, 2020). RNA plays an essential role in biological processes, the link between both the genome and the proteome, among various molecules that could be used to communicate information.

Non-coding RNAs can be used by eukaryotic and prokaryotic cells to transmit signals via extra cellular cables to the target site, where they can be converted into a regulatory response by a detailed molecular apparatus (Leito et al., 2020). Bacterial non-coding RNAs control the expression of host genes, while eukaryotic miRNAs control the expression of bacterial genes (Duval et al., 2016). The replication of SARS-CoV-2 in a human host has been found to be hampered by host miRNA defensive lines (Hosseini and AD, 2020).

Epitranscriptomic alteration is another type of interaction with both commensal bacteria and their host. Difference in the gut microbiota is linked to N6-methyladenosine (m6A) alterations (Jabs et al., 2020). Sometimes, it is essential to allow a drastic change in order to com- 
pletely interrupt the communication between pathogenic bacteria and the host in the pathophysiological process. It's no surprise, then, that faecal transplantation has become one of the most successful therapies in recent years (Aas et al., 2003; Polák et al., 2015). The composition of the microbiome can be modulated to be protected from viral infections, as demonstrated by the probiotic intervention (Yitbarek et al., 2018).

The higher the dose for healthy volunteers of influenza virus, the worse are the symbols of this virus (Memoli et al., 2015). The viral load alone does not, however, clearly forecast the outcome of the disease. Some asymptomatic patients were found to have similar viral loads as patients with COVID-19 symptoms (Lee et al., 2020). In patients with asymptomatic or very low forms of disease, $T$ cell reactions played an important role. Unknowingly, these patients are often the major carriers of the disease (Budden et al., 2017). They demonstrated that in the absence of significant circulating antibodies, a large number of activated/cycling T-cells were functionally complete and specific for SARS-CoV-2 (Sekine et al., 2020). Variations in the intestines and in the lungs are associated with changes in the immune response and the development of the disease (Belkaid and Hand, 2014).

Interleukin IL-6 is one of the substances that is significantly elevated in patients with SARS-CoV-2 disease, particularly in those who do not survive. The removal of this pro-inflammatory interleukin could contribute to patient survival (Zhou et al., 2020).

He et al. (2020b) published an article that directly designates the intestinal microbiota as a potential strategy to fight SARS-CoV-2 infection. However, there is no clear scientific proof that can modulate the intestinal flora and has implications on the prevalence of COVID19 , we argue in the following section that targeting the intestinal microbiota could be a promising pharmacological choice or at the very least an adjuvant treatment of choice. A better and healthier microbiome could be one of the reasons for COVID-19 patients having a lower case fatality ratio. Obesity is one of the strong factors for COVID-19 infection, which has a more severe course (Dietz and SantosBurgoa, 2020). In many cases, we can partially compensate for the lack of symbiotic organism diversity together with fermented foods in our diet, which contain essential probiotics for intestinal health.

\section{Potential mechanisms underlying the role of the gut microbiome in COVID-19 infection}

Coronaviruses are enveloped, single-stranded RNA viruses with a positive single-stranded RNA genome that can infect humans and animals. Coronaviruses and microbiota (particularly the microbiota of the lungs) have close interrelations and they are regulated by viral pathogens in terms of susceptibility to viral infections (Shen et al., 2020). SARS-CoV-2 infection, on the other hand, decreases the expression of ACE2 in the gut tract. As a result, the number of circulating angiogenic cells (CACs) decreases, endangering the endothelium and potentially leading to intestinal dysbiosis (Gheblawi et al., 2020). The lung microbiota can exacerbate infection by altering several factors, including local or systemic inflammatory response, host immune response, mucosal layer protection, and finally unleashing secondary bacterial infections (Shen et al., 2020; Gao et al., 2020).

The viral spike protein of SARS-CoV-2 binds to the cell surface receptor angiotensin converting enzyme 2 and infect the host system.
When virus enters the host, the spike protein is cleaved by a type 2 transmembrane serine protease (TMPRSS2 - an enzyme found in the epithelial cells of the small intestine), which makes the fusion of spike protein into the cell membrane (Matsuyama et al., 2010; Hoffmann et al., 2020; Zhou et al., 2020a) and an additional furin protease enzyme could prime SARS-CoV-2 (Coutard et al., 2020). In enterocytes, the oesophagus, and the lungs, there was a plethora of co-expression of ACE2 and TMPRSS2. High expression of ACE2 and TMPRSS2 in epithelial cells makes the lung, the most susceptible target during coronavirus infection.

ACE2 is a negative regulator of renin-angiotensin-aldosterone system (RAAS) homeostasis, thereby has a key response towards pathology (Gheblawi et al., 2020). ACE2 is a coreceptor for uptake of nutrient and protein absorption in the intestine (Hashimoto et al., 2012). Infection with SARS-CoV-2 causes an inflammatory reaction in the intestine, as noticed by increased calprotectin (biomarker expressed by neutrophils) levels in the faeces (Effenberger et al., 2020) and also the virus entry marks several gastrointestinal disorders. Loss of RAAS homeostasis by ACE2 negatively impacts the inflammatory reactions. Loss of function of ACE2, modifies the $\mathrm{B}(0)$ AT1 amino acid transporter expression and thus reduces the tryptophan microbiota-derived metabolites. This causes an imbalance in the gut homeostasis (Hashimoto et al., 2012; Agus et al., 2018). Reduced expression of antimicrobial peptides and modified intestinal microbial components have been observed in ACE2 knockout mice, which can be restored by administering tryptophan (Hashimoto et al., 2012). Explicit cellular damage caused by viral replication and spread can also play a role in gut epithelial injury and inflammation.

Furthermore, in humans, disturbance of the ACE/ACE2 network during pulmonary hypertension (loss of ACE2) is linked to changes in the gut microbiota (Santisteban et al., 2016; Kim et al., 2020). Reduced availability of ACE2 during SARS-COV-2 infection could be enough to understand its effect on change in gut microbiota's composition, but the effect of SARS-CoV-2 on the metabolic activity of the gut microbiota is also unexplained. Mouse model study involving impaired gut microbiota related with ACE2 deficiency endorses inflammatory responses and imparts susceptibility to colitis, when transmitted to wild-type organisms (Hashimoto et al., 2012). In patients with pre-existing coronary heart disease and other risk factors, a RAAS-ACE2 deficit in COVID-19 can worsen tissue inflammation and lead to more negative COVID-19 outcomes.

Gut microbiota can favour byboosting the immune system, however, dysbiosis can lead to several health ailments. The results of experiments with antibiotic treatment (to deplete the residual microbiota) in the setting of influenza infections have shown that the gut microbiota is critical for regulating viral replication (Ichinohe et al., 2011; Abt et al., 2012; Steed et al., 2017; Bradley et al., 2019). The development of type I interferons and inflammasome-dependent cytokines by pulmonary cells is favoured by bacterial cell wall components and bacterial metabolites (such as desaminotyrosine). Additionally, the gut microbiota improves CD8 + T cell effector activity, which aids in viral clearance (Ichinohe et al., 2011). But treatment with antibiotics makes the infection worse and it can be overcome by using dietary supplements (Trompette et al., 2018). Gut dysfunction-related complications are common in patients with respiratory infections, and they exacerbate the clinical path. Evidence indicates that SARS-CoV-2 infection disrupts the gut-blood barrier, allowing bacteria, endotoxins, and microbial metabolites to spread 
across the body (Wang et al., 2020; Huang et al., 2020; Guan et al., 2020). This could impact the host's initial response to SARS-CoV-2 outbreak, leading to multisystem dysfunction, septic shock, and the systemic cytokine storm that occurs in the second phase of SARSCoV-2 infection, which probably results in death (Guan et al., 2020; Huang et al., 2020; Wang et al., 2020c).

Murine model has shown that the gut microbiota regulates colonic ACE-2 receptors (Yang et al., 2020) and suggesting that it may have a key role in the infectivity as well as the severity of SARS-CoV-2. Various mechanisms of entry have been proposed when considering particular sites of ACE-2 expression inside the intestinal lumen. It has been shown that the ACE-2 receptor in the duodenum increases with age, implying a possible entry mechanism via microbiome interactions (Vuille-dit-Bille et al., 2020). Single-cell transcriptomic study showed a strong expression of ACE2 in oesophageal upper and stratified epithelium and also in enterocytes from the ileum and the colon (Zhang et al., 2020). It was only recently discovered that the gut microbiota plays a part in the extent of viral respiratory tract infections, including those caused by the influenza virus (Bradley et al., 2019). Stromal cells in human lung have been identified as the target of microbiota-driven type 1 interferon (IFN-1) signals, that sets up the defence reaction against early viral load (Steed et al., 2017). Gastrointestinal (GI) symptoms as noted for virus infected patients may be resulted from altered gut microflora composition through IFN-1 mechanism. Noticeably, infection stimulated IFN-1 causes reduction in anaerobic bacteria and enrich the Entero bacteriaceae in the gut environment, resulting to proinflammatory dysbiosis condition (Deriu et al., 2016) and, thus prompting the severity of infection. Adenovirus respiratory infection in a lemur model showed a decrease in abundance of many commensal flora important for a healthy gut microbiome, whereas genera of infectious agents, such as Neisseria, increased (Wasimuddin et al., 2019).

Antigenic presentation to naive $\mathrm{T}$ cells by dendritic cells and antigens derived from commensal species facilitate Treg (regulatory T-cell) cell differentiation, triggering the secretion of anti-inflammatory cytokines (e.g., IL-10) and reinforcing immune responses (Barko et al., 2018; Kamada et al., 2013). Pathogenic bacteria, on the other hand, can elicit pro-inflammatory immune responses; as naive $\mathrm{T}$ cells differentiate into Th1 and Th17 cells. In such circumstances, increased bacterial component translocation across the gut epithelium can occur, resulting in gut epithelial damage and, eventually, disruption of the gut-blood barrier and systemic endotoxemia. These altogether compromises the immune function and an interconnection between dysbiosis, immune cells imbalance and mucosal and systemic inflammation was noticed in Covid-19 patients (Weiss and Henne, 2017).

The gut microbiota was shown to influence lung health through interactions between the gut and the lungs, a phenomenon known as the "gut lung axis" (Pan et al., 2020). Immune cells or the gut microbiota and its metabolites are primarily responsible for possible interactions between cells during the gut-lung axis. Antigen presenting cells phagocytose microbiota and their products that reach the intestinal mucosa, allowing them to be transported to the mesenteric lymph nodes, where they may activate $\mathrm{B}$ and $\mathrm{T}$ cells and these activated cells return to its own site of intestinal mucosa or to another site, for instant, lungs. The second underlying strategy involves transfer of flow of residual bacteria or bacterial products to the lungs through the bloodstream or lymphatic system, resulting in a local or general immunological response that causes further lung damage (Bingula et al., 2017). A study involving mice with lack of gut microbiota showed a lower microbial clearance in the lungs (Fagundes et al., 2012) and the intratracheal lipopolysaccharide (LPS) dosage was found to impair the lung microbiota, which could contribute to disturbance of the intestinal microbiota and an increase in microbial load (Sze et al., 2014). Transition experimental studies with dysbiotic microbiota (Sencio et al., 2020) showed that infections, inflammation, and metabolic disorders can induce dysbiosis, which can alter disease outcomes in distant organs such as the respiratory tract, forming a negative feedback loop.Viral proteins, which vary in structure and fold, can interact with bacterial surfaces such as lipopolysaccharides and peptidoglycans. Microflora has an effect on virus biology both directly and indirectly, and eukaryotic viruses can manipulate bacterial biology (Neu and Mainou, 2020). Surfactin is a cyclic lipopeptide (CLP) that was reported to inhibit $\mathrm{CoV}$ like enveloped viruses (Johnson et al., 2019). Figure 2 shows the potential role of SARS$\mathrm{CoV}-2$ in gut-lung axis dysfunction.

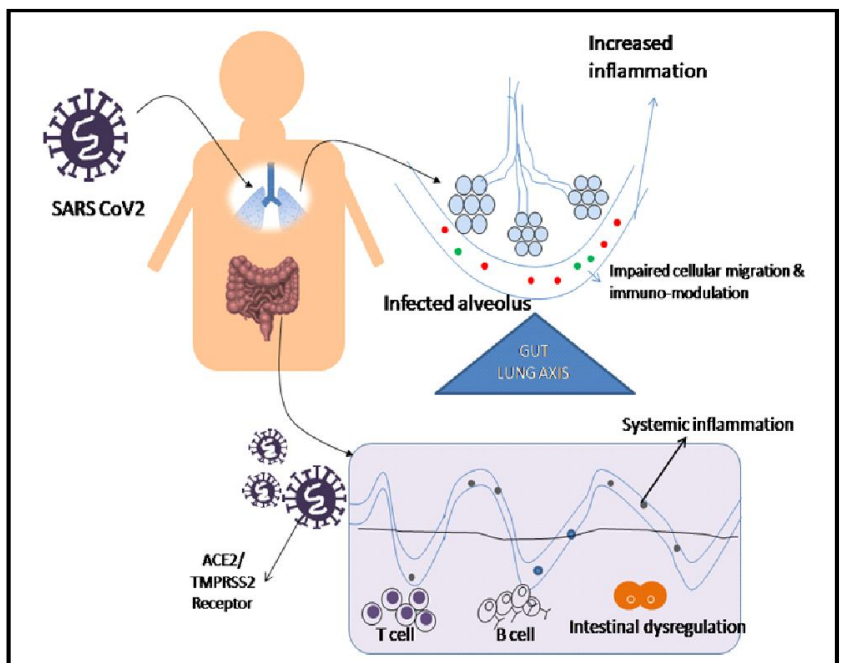

Figure 2: Potential role of SARS-CoV-2 in gut-lung axis dysfunction.

Acute respiratory distress syndrome and pneumonia are two of the most common symptoms of COVID-19. Shen et al. (2020) examined the differences in the composition of the lung microflora in SARSCoV-2-infected patients and showed that the bronchoalveolar lavage fluid (BALF) had varied microbial composition when compared to healthy individual (Shen et al., 2020). Interestingly, a subsection of the core species is associated with the serum levels of proinflammatory cytokines, such as TNF- $\alpha$, IFN- $\gamma$, IL-1 $\beta$, IL-2, IL4, IL-6, IL-8, IL-10, IL-12p70, IL-13.

Furthermore, the risk factors like obesity, high blood pressure, diabetes, age-related and auto-immune disease conditions increases the severity in virus affected patients with disturbances in the gut microbiota, but however, the role of dysbiosis in intestine is need to be elucidated (Badawi et al., 2018; Honce and Schultz-Cherry, 2019). Angiotensin-converting enzyme 2(ACE2), a cell surface receptor involved in dietary amino acid homeostasis, innate immunity, and gut microbial ecology, is increased by a dysbiotic gut environment and epithelial proinflammation; thereby an increased ACE2 creates a potential site for SARS-CoV-2 infection (Wang et al., 2020), from where it can invade to other parts of the body (Zhou et al., 2017). 
Many COVID-19 patients, including those who tested negative by PCR with their respiratory secretions, developed GI infections and had viral RNA detected in their faeces (Xiao et al., 2020). Long-term digestive health issues, especially diarrhoea, are accompanied by changes in gut microbiota composition and diversity (Vandeputte et al., 2016), which are related to immune dysfunction and may explain the late clearance of viral load. This suggests that reforming the gut microbiota could be used as an adjuvant treatment in patients with COVID-19 related digestive symptoms.

In terms of intestinal permeability, a leaky gut exports its microbiota to the lungs, causing pulmonary microbiota to shift. This causes leakage of lipopolysaccharide (LPS) into the circulating system and, thus aggravates the severity of infection by inducing the viral replication and transmission (Robinson and Pfeifer, 2014). This could result in the release of immunosuppressive cytokines, which reduces the immunoregulatory effects of regulatory $\mathrm{T}$ cells and dendritic cells. Both of these modifications cause the virus to evade immune responses and promote viral infection (Anderson, 2020). Some studies reported the activation of NF- $\kappa \mathrm{B}$ signalling by flagellin (microbiota particle that act as damage-associated molecular patterns) toll-like receptor 5 (TLR5) promotes lentiviral pseudovirus binding on lung epithelial cells and that may implicate SARS-CoV-2 infection and lead to harmful inflammatory responses (Golonka et al., 2020).

The human intestine contains a remarkable mechanism for immune system development. Intestinal homeostasis is accomplished by the interaction and synchronisation of innate and adaptive immunity in the gut, which has a mutually beneficial relationship. Antigenpresenting cells, such as dendritic cells in the Peyer's patches of the intestine, Langerhans cells, and macrophages, play a role in the regulation of innate immunity through the gut microbiota (Smythies et al., 2005). These cells have certain immune-tolerance properties against the gut microbiome, similar to that of inflammation anergy by macrophages (Sanos et al., 2009). Mast cells and natural killer cells are two other cells in the innate system that communicate with the gut microbes. The mouse gut microbiota study was found to be critical in expanding the development of IL-22-producing NKp46 cells (Ivanov et al., 2009). Mainly B and T lymphocytes are involved in interaction of gut microbiota and B cells attributed to the gut are typically located in the peyer's patches (Mazmanian et al., 2005). T cells are also essential in the adaptive system and its differentially regulatory T-cells maintain the immune homeostasis of gut lung axis which establishes the anti-inflammatory response (Dhar and Mohanty, 2020). Several microbial metabolites involved in the immune responses, such as Bacteroides fragilis and Clostridia have an impact on cytokine production in the gut, whereby microbiome impairs the interferon signalling and increases the threshold of chronic state protein. According to one theory, COVID-19's interaction with the microbiome can influence cytokine development, possibly leading to an overproduction of pro-inflammatory cytokines like IFNc, MCP1, IP10, and IL1B (Nagano et al., 2012; Rooks et al., 2016), as a result, there's a high probability that COVID-19 can bind with gut microbes, resulting in increased immune cell induction.

Elevated serum levels of lipocalin-2, also regarded as neutrophil gelatinase-associated lipocalin, which is involved in innate immunity, and a greater proportion of $\mathrm{CD} 1 \mathrm{~b}+$ granulocytes were also associated with conventionalization. This research suggests that the gut flora may influence intestinal ACE2 expression and contribute to covid immune dysregulation (Yang et al., 2020). The rise in a blood proteomic risk score (PRS) was linked to a higher risk of developing a clinically serious infection in COVID-19 patients. The C-reactive protein (CRP) are exacerbated during systemic inflammation. The magnitude of COVID-19 can be interconnected to the relationship between PRS and the intestinal microbiome (Gou et al., 2020).

ACE2 deprivation is probably a crucial factor for the negative outcomes in COVID-19 patients with pre-existing comorbidities, and it explores a potential correlation between gut microbiota dysbiosis and the disease conditions. To summarise, the gut microbiota plays a prominent role in host immunity, and SARS-CoV-2 can interfere with the gut microbiota and infect enterocytes, causing gastrointestinal symptoms.

\section{Gut microbiome, immunity and COVID-19}

Coronavirus has infected millions of people and caused many deaths worldwide. The primary site of infection is an airway epithelium where ACE2 receptors, which are necessary for SARS-CoV-2 infection, are in abundance (Fadai et al., 2021). ACE2 receptors are also present in multiple organs including gastrointestinal tracts. ACE2 deficiency causes the modulation of composition of intestinal bacterial population and affects immune system (Chhibber-Goel et al., 2021). Early reports had shown that $2 \%-10 \%$ of COVID-19 patients had gastrointestinal symptoms including diarrhea, but a recent report suggests that about $20 \%$ of the COVID-19 patients has gastrointestinal symptoms. Also, faecal calprotectin, which is responsible for the indication of inflammatory response in gut, was found to be increased in the COVID-19 patients with diarrhea. These evidences indicate that the digestive tract might be an extra pulmonary site for coronavirus infection (Zuo et al., 2021). During COVID-19 infection, the pro-inflammatory cytokines IFN, TNF- $\alpha$, IL- 6 and IL-10 are raised in critical patients. This is termed as 'cytokine storm'. It is notable that some of those cytokines are often correlated with the gut bacterial population. The immune system tries to respond to cytokine storm but affects itself which leads to further multiorgan failure (Morais $e t$ al., 2020; Yazdanpanah et al., 2020). It is also noteworthy that the data from China shows that there is no death of children under age 9 because of COVID-19 cytokine storm, even though children have immature immune system. This shows that cytokine storm will not trigger the immature immune system. However, infants can be severely affected by COVID-19 (Yu, 2021).

The immune system protects the body by employing various mechanisms against invading pathogens. They exhibit three types of immunity. Innate immunity, adaptive immunity and passive immunity. There are two types in passive immunity: natural immunity, which is received from maternal side (breast milk) and artificial immunity, which is received through medicines (Chaplin, 2010; Chowdhury et al., 2020). When a pathogenic microorganism enters a human body, it must get close to the cells to develop infection. The mucous membrane and skin makes this interaction difficult by providing innate immunity (Nicholson, 2016). Adaptive immunity is based on the antigen specific receptor which is expressed on the surface of B lymphocytes and T lymphocytes (Chaplin, 2010). Immune system keeps the body healthy by providing a fine balance between the eradication of invading pathogen and the maintenance of normal healthy tissues. Gut microbial community play a pivotal role in regulating immune system ( $\mathrm{Wu}$ and $\mathrm{Wu}, 2012)$. The human gut microbiota consists of $10^{14}$ microbes including bacteria, virus, fungi and archae, these microorganisms get nourishment from the 
host and in turn regulates the various physiological functions of the host which includes imparting protective immunity against pathogens (Dhar and Mohanty, 2020).

COVID-19 infection affects both the immune system directly and indirectly on (Yazdanpanah et al., 2020). Due to COVID-19 infection around the world, people are experiencing stress and fear about the future consequences of COVID-19. Social distancing is one of the effective methods to prevent the spread of this infection. But, social distancing decreases the access to family and friends which leads to mental stress, anxiety and depression. This indirectly influences on the immune system (Morais et al., 2020). Intestinal microbial population is involved in host metabolism and nutrient absorption and it has a major role in human health. Several studies indicate that intestinal microflora was closely associated with respiratory virus diseases as it occurs through gut-lug axis and it leads to lung damage (Gu et al., 2020). It is observed that gut microbiome composition of COVID-19 patients has altered significantly comparing with healthy individuals even after receiving medications (Yeoh et al., 2021). COVID-19 also causes in depletion of beneficial commensals such as Faecalibacterium prausnitzii (anti-inflammatory bacterium), Bacteroides dorei, Bacteroides massiliensis and Eubacterium ventriosum which correlate with disease severity and down regulates the ACE2 receptors (Morais et al., 2020; Villapol, 2020). A study shows that the significant decrease in the Lachnospiraceae family (Anaerostipes, Fusicatenibacter, E.hallii and Agathobacter) COVID 19 patients comparing with healthy individuals (Gu et al., 2020). Another study conducted with 10 COVID-19 hospitalized patients shows that there are 14 species of gut bacteria are associated with fecal SARS-CoV-2 load across all fecal samples (Villapol, 2020).

Probiotics can help to maintain the gut microbiome by restoring the beneficial microbes and strengthen the immune system against pathogens. Researchers around the world have shown that microorganisms which are having the ability to modulate the immune response could be used to treat the viral and bacterial respiratory infections. The most commonly used probiotic microorganisms are Lactobacillus and Bifidobacterium. Some studies have shown that COVID-19 patients have lower level of Lactobacillus and Bifidobacterium bacterias. These bacterias can be used as probiotics in COVID-19 infection. Probiotic microorganisms are mostly from the gut and can be consumed as isolated form or incorporated in food. Lactobacillus gasseri can be used as probiotic against respiratory viral infection. So, it could possibly use in COVID-19 infection (Morais et al., 2020).

\section{Gut microbiome, diet and COVID-19}

The gut microbiome of healthy adult harbor more than 1000 species of bacteria belonging to few known bacterial phyla (Bacteroidetes and Firmicutes are being the dominant phyla) (Shreiner et al., 2015). COVID-19 can interact with one or more of those 1000 species of gut microbiota (Kalantar-Zadeh et al., 2020). The gut microbiome forms a dynamic environment which can be controlled by dietary habits and probiotic treatments. The amount of food consumed has been reported to shape the microbiome. Several studies shows that proper diet gives an optimal immune response against pathogens including COVID-19 (Hullar and Fu, 2014; Villapol, 2020). The dietary patterns are associated with the differences in the distribution of bacteria and this influences host exposure to microbial metabolites. Dietary intervention studies shows that gut microbiome responses hastily to changes in diet. These changes are often transient (Hullar and Fu, 2014). Besides dietary habits, the gut microbiota is shaped by a combination of extrinsic and intrinsic factors such as medication, lifestyle and host, genetic, metabolic and immune regulations (Leeming et al., 2019). Carbohydrates play a vital role in influencing the gut microbiome (Avila-Nava et al., 2017). It is a known fact that the modern Western diet which contains processed, canned and frozen foods has less fiber content comparing with the diet consumed by developing countries like India. It has also observed that the Western diet tends to increase in the abundance of bacteria belonging to Firmicutes phylum with the simultaneous drastic decrease in the bacteria of Bacteroidetes phylum (Senghor et al., 2018). Protein intake is essential for the production of antibodies (Villapol, 2020).

Children in Africa are reported to have high Bacteroidetes population and low Firmicutes population in the gut as their diet include highfiber content. It was also observed that increased short chain fatty acids (SCFA) in their feces when compared to the children of European origin. SCFAs are important in immunoregulation and it alleviates type 2 diabetes mellitus (De Filippo et al., 2017; Rishi et al., 2020; $\mathrm{Yu}, 2021)$. Plant-based high fiber diet was found to elevate the Bifidobacterium and reduces the Firmicutes to Bacteroidetes ratio (Avila-Nava et al., 2017). A study observed in India shows that Prevotella are the dominant species in the gut of North-central Indians whose diet mostly contains carbohydrate rich plant-based food. Whereas, it has been observed that Fecalibacteria, Ruminococci and Bacteroidetes species of bacteria are dominant in the gut of South Indians who majorly consume omnivorous diet (Dhakan et al., 2019). During COVID-19 pandemic till $31^{\text {st }}$ May 2020 in India, it was observed that the fatality rate in India was the lowest in comparison with the Western countries. It may be inferred that plant-based, fiber rich, home cooked diet during lockdown in India might increase the population of symbiotic microflora, which is resulted in eliciting anti-inflammatory responses and production of SCFAs (Rishi et al., 2020).

Hairy morphology of the intestine can be maintained by branchedchain amino acids and these amino acids help to increase intestinal immunoglobulin levels which improves intestinal barrier. High protein intake improves anti-inflammatory property. Omega 3 fatty acids are the excellent source of anti-inflammatory and antioxidant capacity (Villapol, 2020). The dietary intake of iron, zinc and selenium promotes immunity to fight against COVID-19 (Rishi et al.,2020). Food sources of fiber (example: whole grains) are suggested to have a prebiotic-like effect on the gut microbiome. Along with fiber, the dietary intake of polyphenols, magnesium, vitamin C, vitamin D and vitamin $\mathrm{E}$ exert a beneficial effect on microbiota composition. Polyphenols are found higher levels in foods such as vegetables, cereals, tea, coffee and wine (Leeming et al., 2019). Foods such as dark chocolate, whole greens, black beans and avocados are some of the good dietary sources of magnesium. soaked almonds, sunflower seeds, hazel nuts and peanut butter are the major sources of vitamin E. vitamin $\mathrm{C}$ rich foods include lemon, spinach and broccoli. Egg and fish are some sources of Vitamin D (Arshad et al., 2020; Rishi et al., 2020).

\section{The gut microbiome and suggested treatments for COVID-19 infection}

Parallel to attempts to understand the pathogenesis of COVID-19 disease, the pandemic has caused an unprecedented number of 
investigational treatment trials to be developed and initiated. Over 3800 clinical trials targeting COVID-19 have been reported around the world as of October 2020. The gut microbiome has been shown to modulate a broad variety of therapeutic compounds used in clinical practice, including antivirals, antihypertensives, and antidiabetic drugs, and baseline gut microbiome profiles can predict cancer therapy responses. It is clear that inter-individual variation in gut microbiome will affect the effectiveness of COVID-19 treatments (Zhang et al., 2020). To combat COVID-19, a variety of drugs are being tested, with some showing signs of interaction with the gut microbiome. Azithromycin, a macrolide antibiotic, is being studied extensively as a COVID-19 treatment, primarily in conjunction with hydroxychloroquine. The action of azithromycin on gut bacteria is well known, therefore, it is used to treat Campylobacter disease in many regions of the world. A 3-day course of azithromycin $(10 \mathrm{mg} / \mathrm{kg})$ was shown to reduce alpha diversity of the gut microbiota in a blinded, randomized, placebo-controlled study in young children, with loss of the genus bifidobacterium at 14 days (Vikse and Henry, 2020).

Furthermore, functional study of the metagenomes of African children treated with azithromycin revealed that metabolic pathways involved in immune function and inflammation were under represented (Strangfeld et al., 2017). Antibiotic use has been common in COVID-19 patients, and there is increasing concern that antimicrobial resistance could improve. Antibiotic use prior to viral exposure has also been shown to predispose people to more serious respiratory infections. Animals given antibiotics had an abrogated interferon signature in the lung stroma, allowing early virus replication in the epithelia in a mouse model of influenza infection. Interestingly, faecal transplant following antibiotics recovered the interferon signature, indicating that the gut microbiome plays a significant role in deciding the integrity of barrier defenses against disease at sites distant to the GI tract (Barbara et al.,2017). If repeated in humans, this model may be appropriate to other viruses which enter through the respiratory epithelium, including SARS-CoV-2. As a result, a proposal has been made for intervention studies involving gut microbiota manipulation, such as probiotics and faecal transplantwith the goal of preventing or limiting the clinical extent of COVID19 infection. Tocilizumab, a monoclonal anti-IL6 antibody, is the subject of several studies aimed at reducing the hyperinflammatory cytokine release storm seen in some COVID-19 patients (Hu et al., 2020). Concern was raised in some areas that tocilizumab, a drug to treat rheumatological conditions like rheumatoid arthritis, is proven to cause small gastrointestinal perforation (2-3 per 1000 patients). While patients with diverticular disease are thought to be at increased risk, the mechanism is unidentified. The existence of diverticular disease, regardless of effects, is linked to a microbial imbalance. It is important to note that IL-6-deficient mice have such a thinning mucus gel layer and a compromised gut-epithelial barrier. Bacteroidales bacteria promote intra-epithelial lymphocytes in the colon that generate IL-6, increasing the likelihood that the gut microbiota's composition or role is involved in the aetiology of tocilizumabrelated perforation (Musa, 2020).

Eventually, there is clearly a massive clinical research need for such a vaccine against COVID-19, and a number of trials are currently in progress globally. Immune responses to vaccine management against viral and other pathogens are well known to vary significantly between patients; this variability may reflect the interaction of a variety of factors, including the compositionand/or functionality of the gut microbiome (Guan et al., 2020). Although, there is a lot of evidence from animal research that suggests immune response heterogeneity to vaccines, potential human data shows that antibioticmediated gut microbiome disturbance impairs the immune reaction to influenza vaccination in patients who have low pre-existing antiinfluenza antibody titres. As a result, when recruiting participants for COVID-19 vaccine studies, recent use of antibiotics (or other factors that may disturb the gut microbiome) may be important to consider (Jin et al., 2020).

\section{Conclusion}

The diversity of the gut microbiota and the existence of beneficial microorganisms in the gut can play a key role in the progression of this disease.The gut microbiome is essential for human health and disease, and it may be important in the interaction between COVID19 infection and the host. Microbiome research may aid our understanding of the pandemic and provide insight into prevention and treatment options. The long-term effects of COVID-19 infection on a number of organs are unclear at this time, although there is the possibility of long-term effects. The gut microbiota can affect immune response and, therefore disease progression. In COVID-19, both an overactive and underactive immune response, likely linked to gut microbiota status, may result in severe clinical complications. The unhealthiness of microbiota may then be a still-underappreciated risk factor. Since sufficient, effective, and inexpensive prebiotics and probiotics may help microbiota, their use should be regarded as an adjunctive treatment to reduce COVID-19 progression in infected individuals, or as a prevention measure for non-infected people at risk during COVID-19 spread. As we understand more about virus, it will be vital to understand the pandemic's impact on the gut microbiota, as well as the possible long-term consequences.

\section{Conflict of interest}

The authors declare that there are no conflicts of interest relevant to this article.

\section{References}

Abt, M.C.; Osborne, L.C., Monticelli, L.A.; Doering, T.A.; Alenghat, T.; Sonnenberg, G.F.; Paley, M.A.; Antenus, M.; Williams, K.L.; Erikson, J.; Wherry, E. J and Artis, D. (2012). Commensal bacteria calibrate the activation threshold of innate antiviral immunity. Immunity. 37(1):158-70.

Agus, A.; Planchais, J and Sokol, H. (2018). Gut microbiota regulation of tryptophan metabolism in health and disease. Cell Host Microbe, 23:716-724

Anderson, G. (2020). Psychological stress and Covid-19: Interactions with gut microbiome and circadian rhythm in driving symptom severity. Preprint, London: CRC Scotland and London.

Arshad, M.S.; Khan, U.; Sadiq, A.; Khalid, W.; Hussain, M.; Yasmeen, A.; Asghar, $\mathbf{Z}$ and Rehana, H. (2020). Coronavirus disease (COVID 19) and immunity booster green foods: A mini review. Food Sci. Nutr., 8(8):39713976 .

Avila Nava, A.; Noriega, L.G.; Tovar, A.R.; Granados, O.; Perez Cruz, C.; Pedraza Chaverri, J and Torres, N. (2017). Food combination based on a pre hispanic Mexican diet decreases metabolic and cognitive abnormalities and gut microbiota dysbiosis caused by a sucrose enriched high fat diet in rats. Mol. Nutr. Food. Res., 61(1):1501-15023.

Badawi, A.; Velummailum, R.; Ryoo, S.G.; Senthinathan, A.; Yaghoubi, S.; Vasileva, D.; Ostermeier, E.; Plishka, M.; Soosaipillai, M and Arora, P. (2018). Prevalence of chronic comorbidities in dengue fever and West Nile virus: A systematic review and meta-analysis. PLoS ONE., 13(7): 0200200 . 
Barbara, G.; Scaioli, E.; Barbaro, M.R.; Biagi, E.; Laghi, L.; Cremon, C.; Marasco, G.; Colecchia, A.; Picone, G.; Salfi, N and Capozzi, F. (2017). Gu microbiota, metabolome and immune signatures in patients with uncomplicated diverticular disease. Gut., 66(7):1252-1261.

Barko, P.C.; McMichael, M.A.; Swanson, K.S and Williams, D.A. (2018). The gastrointestinal microbiome: A review J. Vet. Intern. Med., 32:925 .

Baud, D.; Dimopoulou Agri, V.; Gibson, G.R.; Reid, G and Giannoni, E. (2020) Using probiotics to flatten the curve of coronavirus disease COVID 2019 pandemic. Front. Public Health, 8:186.

Bell, A.; Brunt, J.; Crost, E.; Vaux, L and Nepravishta, R. (2019). Elucidation of a sialic acid metabolism pathway in mucus-foraging Ruminococcus gnavus unravels mechanisms of bacterial adaptation to the gut. Nat. Microbiol., 4:2393-2404.

Bingula, R.; Filaire, M.; Radosevic-Robin, N.; Bey, M.; Berthon, J.Y.; BernalierDonadille, A.; Vasson, M.P and Filaire, E. (2017). Desired turbulence? Gut-Lung Axis, immunity, and lung cancer. J. Oncol., 15: e5035371.

Bradley, K.C.; Finsterbusch, K.; Schnepf, D.; Crotta, S.; Llorian, M.; Davidson, S.; Fuchs, S.Y.; Staeheli, P and Wack, A. (2019). Microbiota-driven tonic interferon signals in lung stromal cells protect from influenza virus infection. Cell Rep., 28:245-256.

Cai, Q.; Chen, F.; Wang, T.; Luo, F.; Liu, X.; Wu, Q.; He, Q.; Wang, Z.; Liu, Y.; Liu, $\mathbf{L}$ and Chen, J. (2020). Obesity and COVID-19 severity in a designated hospital in Shenzhen, China. Diabetes Care, 43(7):1392-1398.

Cani, P.D (2018). Human gut microbiome: Hopes, threats and promises. Gut., 67:1716-1725.

Chaplin, D.D (2010). Overview of the immune response. J. Allergy Clin Immunol., 125(2):S3-S23.

Chhibber-Goel, J.; Gopinathan, S and Sharma, A. (2021). Interplay between severities of COVID-19 and the gut microbiome: Implications of bacterial co-infections? Gut. Pathog., 13(1):1-6.

Chowdhury, M.A.; Hossain, N.; Kashem, M.A.; Shahid, M.A and Alam, A. (2020). Immune response in COVID-19: A review. J. Infect. Public Health, 13(11): 1619-1629.

Coutard, B.; Valle, C.; de Lamballerie, X.; Canard, B.; Seidah, N.G and Decroly, E. (2020). The spike glycoprotein of the new coronavirus 2019nCoV contains a furin-like cleavage site absent in $\mathrm{CoV}$ of the same clade. Antivir. Res., 176:104742-104746.

De Filippo, C.; Di Paola, M.; Ramazzotti, M.; Albanese, D.; Pieraccini, G.; Banci, E.; Miglietta, F.; Cavalieri, D and Lionetti, P. (2017). Diet, environments, and gut microbiota. A preliminary investigation in children living in rural and urban Burkina Faso and Italy. Front. Microbiol., 8: 1979.

Deriu, E.; Boxx, GM.; He, X.; Pan, C.; Benavidez, S.D.; Cen, L.; Rozengurt, N.; Shi, W and Cheng, G. (2016). Influenza virus affects intestinal microbiota and secondary Salmonella infection in the gut through Type I interferons. PLoS Pathog., 12:e1005572.

Dhakan, D.B.; Maji, A.; Sharma,A.K.; Saxena, R.; Pulikkan, J.; Grace, T.; Gomez, A.; Scaria, J.; Amato, K.R and Sharma, V.K. (2019). The unique composition of Indian gut microbiome, gene catalogue, and associated fecal metabolome deciphered using multiomics approaches Gigascience, 8(3): giz004.

Dhar, D. and Mohanty, A. (2020). Gut microbiota and COVID-19-possible link and implications. Virus Res., 285:198018.

Doan, T.; Hinterwirth, A.; Worden, L.; Arzika, A.M.; Maliki, R.; Abdou, A.; Kane, S.; Zhong, L.; Cummings, S.L.; Sakar, S and Chen, C. (2019). Gut microbiome alteration in MORDOR I: A community-randomized trial of mass azithromycin distribution. Nat. Med., 25(9):13701376.
Effenberger, M.; Grabherr, F; Mayr, L; Schwaerzler, J.; Nairz, M.; Seifert, M.; Hilbe, R.; Seiwald, S.; Scholl-Buergi, S.; Fritsche, G and Bellmann-Weiler, R. (2020). Faecal calprotectin indicates intestinal inflammation in COVID-19. Gut., 69(8):1543-1544.

Elsayed, S. and Zhang, K. (2004). Human infection caused by Clostridium hathewayi. Emerg. Infect. Dis., 10:1950.

Esposito, S.; Polinori, I and Rigante, D. (2019). The gut microbiota-host partnership as a potential driver of Kawasaki syndrome. Front Pediatr., 7:124.

Fadai, N.T.; Sachak-Patwa, R.; Byrne, H.M.; Maini,P.K.; Bafadhel, M and Nicolau Jr, D.V. (2021). Infection, inflammation and intervention: mechanistic modelling of epithelial cells in COVID-19. J. R. Soc. Interface, 18(175):20200950.

Fagundes, C.T.; Amaral, F.A.; Vieira, A.T.; Soares, A.C.; Pinho, V.; Nicoli, J.R.; Vieira, L.Q.; Teixeira, M.M and Souza, D. G. (2012). Transient TLR activation restores inflammatory response and ability to control pulmonary bacterial infection in germfree mice. J. Immunol., 188(3):1411-1420.

Fang, L.; Karakiulakis, G and Roth, M. (2020). Are patients with hypertension and diabetes mellitus at increased risk for COVID-19 infection. The Lancet. Respir. Med., 8(4):21.

Gao, Q.Y.; Chen, Y.X and Fang, J.Y. (2019). Novel coronavirus infection and gastrointestinal tract. J. Dig. Dis., 21(3):125-136.

Garmaeva, S.; Sinha, T.; Kurilshikov, A.; Fu, J.; Wijmenga, C and Zhernakova, A. (2019). Studying the gut virome in the metagenomic era: Challenges and perspectives. BMC Biol., 17:84.

Geva-Zatorsky, N.; Sefik, E.; Kua, L.; Pasman, L and Tan, T.G. (2017). Mining the human gut microbiota for immunomodulatory organisms. Cell, 168:928-943.

Gheblawi, M.; Wang, K.; Viveiros, A.; Nguyen, Q.; Zhong, J.C.; Turner, A.J.; Raizada, M.K.; Grant, M.B and Oudit, G.Y. (2020). Angiotensinconverting enzyme 2: SARS-CoV-2 receptor and regulator of the renin-angiotensin system: Celebrating the 20th anniversary of the discovery of ACE2. Circ. Res., 126:1456-1474.

Golonka, R. M.; Saha, P.; Yeoh, B.S.; Chattopadhay, S.; Gewirtz, A.T.; Joe, B and Vijay-kumar, M. (2020). Harnessing innate immunity to eliminate SARS-CoV-2 and ameliorate COVID-19 disease. Physiol Genomics, 52(5):217-221.

Gou, W.; Fu, Y.; Yue, L.; Chen, G.D.; Cai, X.; Shuai, M.; Xu, F.; Chen, H.; Zhu, Y.; Xiao, M. L.; Jiang, Z.; Miao, Z.; Xiao, C.; Shen, B.; Wu, X.; Zhao, H.; Ling, W.; Wang, J.; Chen, Y.M.; Guo, T and Zheng, J.S. (2020). Gut microbiota may underlie the predisposition of healthy individuals to COVID-19. medRxiv.

Gu, S.; Chen, Y.; Wu, Z.; Chen, Y.; Gao, H.; Lv, L.; Guo, F.; Zhang, X.; Luo, R.; Huang, C and Lu, H. (2020). Alterations of the gut microbiota in patients with COVID-19 or H1N1 influenza. Clin Infect Dis., 71(10): 2669-2678.

Guan, W. J.; Ni, Z. Y.; Hu, Y.; Liang, W. H.; Ou, C. Q.; He, J. X.; Liu, L.; Shan, H.; Lei, C. L.; Hui, D.S.C.; Du, B.; Li, L.J.; Zeng, G.; Yuen, K.Y.; Chen, R.C.; Tang, C.L.; Wang, T.; Chen, P.Y.; Xiang, J.; Li, S. Y.; Wang, J. L.; Liang, Z. J.; Peng, Y. X.; Wei, L.; Liu, Y.; Hu, Y. H.; Peng, P.; Wang, J. M.; Liu, J. Y.; Chen, Z.; Li, G.; Zheng, Z. J.; Qiu, S. Q.; Luo, J.; Ye, C. J.; Zhu, S. Y and Zhong, N. S. (2020). Clinical characteristics of coronavirus disease 2019 in China. N. Engl. J. Med., 382: 1708-1720.

Hagan, T.; Cortese, M.; Rouphael, N.; Boudreau, C.; Linde, C.; Maddur, M.S.; Das, J.; Wang, H.; Guthmiller, J.; Zheng, N.Y and Huang, M. (2019). Antibiotics-driven gut microbiome perturbation alters immunity to vaccines in humans. Cell, 178(6):1313-1328.

Han, Y.; Jia, Z.; Shi, J.; Wang, W and He, K. (2020). The active lung microbiota landscape of COVID-19 patients. medRxiv. 
Hashimoto, T.; Perlot, T.; Rehman, A.; Trichereau, J.; Ishiguro, H., Paolino, M., Sigl, V.; Hanada, T.; Hanada, R.; Lipinski, S. and Wild, B. (2012). ACE2 links amino acid malnutrition to microbial ecology and intestinal inflammation. Nature, 487:477-481.

Henke, M.T.; Kenny, D.J.; Cassilly, C.D.; Vlamakis, H and Xavier, R.J. (2019) Ruminococcus gnavus, a member of the human gut microbiome associated with Crohn's disease, produces an inflammatory polysaccharide. Proc. Natl. Acad. Sci., 116:12672-12677.

Hill, M.A.; Mantzoros, C and Sowers, J.R. (2020). Commentary: COVID-19 in patients with diabetes. Metabolism, 107:154-217.

Hoffmann, M.; Kleine-Weber, H.; Schroeder, S.; Krüger, N.; Herrler, T.; Erichsen, S.; Schiergens, T. S.; Herrler, G.; Wu, N. H.; Nitsche, A.; Müller, M. A.; Drosten, C and Pöhlmann, S. (2020). SARS-CoV-2 cell entry depends on ACE2 and TMPRSS2 and is blocked by a clinically proven protease inhibitor. Cell, 181(2):271-280.

Honce, R. and Schultz-Cherry, S. (2019). Impact of obesity on influenza A Virus pathogenesis, immune response, and evolution. Front. Immunol., 10:1071-1084.

Hu, Y.; Xie, G.; Jiang, X.; Shao, K.; Tang, X and Gao, G. (2020). The relationships between the free-living and particle-attached bacterial communities in response to elevated eutrophication. Front. Microbiol., 11:423.

Huang, C.; Wang,Y.; Li, X.; Ren, L.; Zhao, J.; Hu, Y.; Thang, L.; Fan, G; Xu, J.; Gu, X.; Cheng, Z.; Yu, T.; Xia, J.; Wei, Y.; Wu, W.; Xie, X.; Yin, W.; Li, H.; Liu, M.; Xiao, Y.; Gao, H.; Guo, L.; Xie, J.; Wang, G.; Jiang, R.; Gao, Z.; Jin, Q.; Wang, J and Cao, B. (2020). Clinical features of patients infected with 2019 novel coronavirus in Wuhan, China. Lancet., 395(10223):497-506.

Hugon, P.; Dufour, J.C.; Colson, P.; Fournier, P.E.; Sallah, K. and Raoult, D. (2015) A comprehensive repertoire of prokaryotic species identified in human beings. Lancet Infect. Dis., 15:1211-1219.

Hullar, M.A. and Fu, B.C. (2014). Diet, the gut microbiome, and epigenetics. Cancer J. (Sudbury, Mass.), 20(3):170.

Ichinohe, T.; Pang, I.K.; Kumamoto, Y.; Peaper, D.R.; Ho, J.H.; Murray, T.S and Iwasaki, A. (2011). Microbiota regulates immune defense against respiratory tract influenza A virus infection. Proc. Natl. Acad. Sci., 108:5354-5359.

Ivanov, I. I.; Atarashi, K.; Manel, N.; Brodie, E. L.; Shima, T.; Karaoz, U.; Wei, D.; Goldfarb, K. C.; Santee, C. A.; Lynch, S. V.; Tanoue, T.; Imaoka, A.; Itoh, K.; Takeda, K.; Umesaki, Y.; Honda, K and Littman, D. R. (2009). Induction of intestinal Th17 cells by segmented filamentous bacteria. Cell, 139(3):485-498.

Jin, X.; Lian, J.S.; Hu, J.H.; Gao, J.; Zheng, L.; Zhang, Y.M.; Hao, S.R.; Jia, H.Y.; Cai, H.; Zhang, X.L and Yu, G.D. (2020). Epidemiological, clinical and virological characteristics of 74 cases of coronavirus-infected disease 2019 (COVID-19) with gastrointestinal symptoms. Gut., 69(6):1002-1009.

Johnson, B. A.; Hage, A.; Kalveram, B.; Mears, M.; Plante, J. A and Rodriguez, S. E. (2019). Peptidoglycan-associated cyclic lipopeptide disrupts viral infectivity. J. Virol., 93:e01282-e01219.

Jones, V.G.; Mills, M.; Suarez, D.; Hogan, C.A.; Yeh, D.; Segal, J.B.; Nguyen, E.L.; Barsh, G.R.; Maskatia, S and Mathew R. (2020). COVID-19 and Kawasaki disease: Novel virus and novel case. Hosp Pediatr., 10:537-540.

Kalantar-Zadeh, K.; Ward, S.A.; Kalantar-Zadeh, K and El-Omar, E.M. (2020). Considering the effects of microbiome and diet on SARS-CoV-2 infection: Nanotechnology Roles. ACS Nano., 14:5179-5182.

Kamada, N.; Seo, S.U.; Chen, G.Y and Nunez, G. (2013). Role of the gut microbiota in immunity and inflammatory disease. Nat. Rev. Immunol., 13:321-335.
Kim, S.; Rigatto, K.; Gazzana, M.B.; Knorst, M.M.; Richards, E.M.; Pepine, C.J and Raizada, M.K. (2020). Altered gut microbiome profile in patients with pulmonary arterial hypertension. Hypertension, 75:10631071 .

Kuba, K.; Imai, Y.; Rao, S.; Gao, H.; Guo, F.; Guan, B.; Huan, Y.; Yang, P.; Zhang, Y.; Deng, W and Bao, L. (2005). A crucial role of angiotensin converting enzyme 2 (ACE2) in SARS coronavirus-induced lung injury. Nat Med., 11(8):875-879.

Leeming, E.R.; Johnson, A.J.; Spector, T.D and Le Roy, C.I. (2019). Effect of diet on the gut microbiota: Rethinking intervention duration. Nutrients, 11(12):2862.

Li, J.; Jia, H.; Cai, X.; Zhong, H.; Feng, Q.; Sunagawa, S.; Arumugam, M.; Kultima, J.R.; Prifti, E.; Nielsen, T.; Juncker, A.S.; Manichanh, C.; Chen, B.; Zhang, W.; Levenez, F.; Wang, J.; Xu, X.; Xiao, L.; Liang, S.; Zhang, D.; Zhang, Z.; Chen, W.; Zhao, H.; Al-Aama, J.Y.; Edris, S.; Yang, H.; Wang, J.; Hansen, T.; Nielsen, H.B.; Brunak, S.; Kristiansen, K.; Guarner, F.; Pedersen, O.; Doré, $\mathbf{J}$ and Ehrlich, S.D. (2014). An integrated catalog of reference genes in the human gut microbiome. Nat. Biotechnol., 32:834-841.

Li, N.; Ma, W.T.; Pang, M.; Fan, Q.L and Hua, J.L. (2019). The commensal microbiota and viral infection: A comprehensive review. Front Immunol., 10:15-51.

Lie'vin-Le Moal, V. and Servin, A.L. (2006). The front line of enteric hos defense against unwelcome intrusion of harmful microorganisms: Mucins, antimicrobial peptides, and microbiota. Clin. Microbiol. Rev., 19(2):315-37.

Matson, V.; Fessler, J.; Bao, R.; Chongsuwat, T.; Zha, Y.; Alegre, M.L.; Luke, J.J and Gajewski, T.F. (2018). The commensal microbiome is associated with anti-PD-1 efficacy in metastatic melanoma patients. Science., 359(6371):104-108.

Matsuyama, S.; Nagata, N.; Shirato, K.; Kawase, M.; Takeda, M and Taguchi, F. (2010). Efficient activation of the severe acute respiratory syndrome coronavirus spike protein by the transmembrane protease TMPRSS2. J. Virol., 84:12658-12664.

Mazmanian, S.K.; Liu, C.H; Tzianabos, A.O and Kasper, D.L. (2005). An immunomodulatory molecule of symbiotic bacteria directs maturation of the host immune system. Cell, 122(1):107-118.

McIlroy, J.R.; Mullish, B.H. Goldenberg, S.D.; Ianiro, G and Marchesi, J.R. (2020). Intestinal microbiome transfer, a novel therapeutic strategy for COVID-19 induced hyperinflammation?: In reply to, 'COVID19: Immunology and treatment options', Clinical Immunology (Orlando, Fla.)., 218:10852.

Mendes, V.; Galvao, I and Vieira, A.T. (2019). Mechanisms by which the gut microbiota influences cytokine production and modulates host inflammatory responses. J. Interferon Cytokine Res., 39(7):393409.

Morais, A.H.; Passos, T.S.; Maciel, B.L and da Silva-Maia, J.K. (2020). Can probiotics and diet promote beneficial immune modulation and purine control in coronavirus infection?. Nutrients, 12(6):1737.

Morais, A.H.A.; Aquino, J.S.; da Silva-Maia, J.K.; Vale, S.H.L.; Maciel, B.L.L and Passos, T.S. (2020). Nutritional status, diet and viral respiratory infections: Perspectives for severe acute respiratory syndrome coronavirus 2. Br. J. Nutr., pp:1-12.

Musa, S. (2020). Hepatic and gastrointestinal involvement in coronavirus disease 2019 (COVID-19): What do we know till now?. Arab J. Gastroenterol., 21(1):3-8.

Nagano, Y.; Itoh, K and Honda, K. (2012). The induction of treg cells by gut-indigenous clostridium. Curr. Opin. Immunol., 24(4):392-397.

Neu, U. and Mainou, B. A. (2020). Virus interactions with bacteria: Partners in the infectious dance. PLoS Pathog., 16:1008234. 
Ng, S.C. and Tilg, H. (2020). COVID-19 and the gastrointestinal tract: more than meets the eye. Gut., 69:973-4.

Nicholson, L.B. (2016). The immune system. Essays Biochem, 60(3):275301 .

Pan, L.; Mu, M.; Yang, P.; Sun, Y.; Wang, R.; Yan, J.; Li, P.; Hu, B.; Wang, J.; Hu, C.; Jin, Y.; Niu, X.; Ping, R.; Du, Y.; Li, T.; Xu, G.; Hu, Q and Tu, L. (2020). Clinical characteristics of COVID-19 patients with digestive symptoms in Hubei, China: A descriptive, cross-sectional, multicenter study. Am. J. Gastroenterol., 115(5):766-773.

Png, C. W.; Linden, S.K.; Gilshenan, K.S.; Zoetendal, E.G and McSweeney, C.S (2010). Mucolytic bacteria with increased prevalence in IBD mucosa augment in vitro utilization of mucin by other bacteria. Am. J. Gastroenterol., 105:2420-2428.

Rishi, P.; Thakur, K.; Vij, S.; Rishi, L.; Singh, A.; Kaur, I.P.; Patel, S.K.; Lee, J.K and Kalia, V.C. (2020). Diet, gut microbiota and COVID-19. Indian J. Microbiol., pp:1-10.

Robinson, C.M and Pfeifer, J.K. (2014). Viruses and the microbiota. Annu. Rev. Virol., 1:55-69.

Rooks, M.G and Garrett, W.S. (2016). Gut microbiota, metabolites and host immunity. Nat Rev Immunol., 16(6):341-52.

Sanos, S.L.; Bui, V.L.; Mortha,A.; Oberle, K.; Heners, C.; Johner, C and Diefenbach, A. (2009). ROR gammat and commensal microflora are required for the differentiation of mucosal interleukin 22-producing NKp46+ cells. Nat Immunol., 10:83-91.

Santisteban, M.M.; Kim, S.; Pepine, C.J and Raizada, M.K. (2016). Brain-gut bone marrow axis: Implications for hypertension and related therapeutics. Circ. Res., 118:1327-1336.

Scarpellini, E.; Ianiro, G.; Attili, F.; Bassanelli, C.; De Santis, A and Gasbarrini, A. (2015). The human gut microbiota and virome: Potential therapeutic implications. Dig. Liver Dis., 47:1007-1012.

Sencio, V.; Barthelemy, A.; Tavares, L. P.; Machado, M. G.; Soulard, D.; Cuinat C.; Queiroz-Junior, C.M.; Noordine, M.L.; Salome-Desnoulez, S.; Deryuter, L.; Foligné, B.; Wahl, C.; Frisch, B.; Vieira, A. T.; Paget, C.; Milligan, G.; Ulven, T.; Wolowczuk, I.; Faveeuw, C.; Le Goffic, R.; Thomas, M.; Ferreira, S.; Teixeira, M. M and Trottein, F. (2020). Gut dysbiosis during influenza contributes to pulmonary pneumococcal superinfection through altered short-chain fatty acid production. Cell Rep., 30:29342947

Senghor, B.; Sokhna, C.; Ruimy, R and Lagier, J.C. (2018). Gut microbiota diversity according to dietary habits and geographical provenance. Hum. Microbiome J., 7:1-9.

Shen, T. D. (2017). Diet and gut microbiota in health and disease. Nestle Nutr. Inst. Workshop Ser., 88:117-126.

Shen, Z.; Xiao, Y.; Kang, L.; Ma, W.; Shi, L.; Zhang, L.; Zhou, Z.; Yang, J.; Zhong, J.; Yang, D and Guo, L. (2020). Genomic diversity of SARS-CoV-2 in coronavirus disease 2019 patients. Clin. Infect. Dis., 71:713-720.

Shreiner, A.B.; Kao, J.Y and Young, V.B. (2015). The gut microbiome in health and in disease. Curr. Opin. Gastroenterol., 31:69.

Smythies, L.E.; Sellers, M.; Clements, R.H.; Mosteller-Barnum, M.; Meng, G.; Benjamin, W.H.; Orenstein, J.M and Smith, P.D. (2005). Human intestina macrophages display profound inflammatory anergy despite avid phagocytic and bacteriocidal activity. J. Clin. Invest., 115:66-75.

Steed, A.L.; Christophi, G.P.; Kaiko, G.E.; Sun, L.; Goodwin, V.M.; Jain, U.; Esaulova, E.; Artyomov, M.N.; Morales, D.J.; Holtzman, M.J.; Boon, A.C.M.; Lenschow, D.J and Stappenbeck, T.S. (2017). The microbial metabolite desaminotyrosine protects from influenza through type I interferon. Science, 357:498-502.
Strangfeld, A.; Richter, A.; Siegmund, B.; Herzer, P.; Rockwitz, K.; Demary, W.; Aringer, M.; Meißner, Y.; Zink, A and Listing, J. (2017). Risk for lower intestinal perforations in patients with rheumatoid arthritis treated with tocilizumab in comparison to treatment with other biologic or conventional synthetic DMARDs. Ann. Rheum. Dis., 76(3): 504-510.

Strathdee, S.A.; Davies, S.C and Marcelin, J.R. (2020). Confronting antimicrobial resistance beyond the COVID-19 pandemic and the 2020 US election. The Lancet, 396(10257):1050-1053.

Sze, M.A.; Tsuruta, M.; Yang, S.W.; Oh, Y.; Man, S.F.; Hogg, J.C and Sin D.D. (2014). Changes in the bacterial microbiota in gut, blood, and lungs following acute LPS instillation into mice lungs. PLoS ONE., 9(10): 111228 .

Trompette, A.; Gollwitzer, E.S.; Pattaroni, C.; Lopez-Mejia, I.C.; Riva, E.; Pernot, J.; Ubags, N.; Fajas, L.; Nicod, L.P and Marsland, B.J. (2018). Dietary fiber confers protection against flu by shaping Ly6c" patrolling monocyte hematopoiesis and CD8 + T. Cell Metabolism, Immunity, 48(5):992-1005.

Vandeputte, D.; Falony, G.; Vieira-Silva, S.; Tito, R.Y.; Joossens, M and Raes, J. (2016). Stool consistency is strongly associated with gut microbiota richness and composition, enterotypes and bacterial growth rates. Gut., 65:57-62.

Vikse, J. and Henry, B.M. (2020). Tocilizumab in COVID-19: Beware the risk of intestinal perforation. Int. J. Antimicrob. Agents, 56(1): 106-009.

Villapol, S. (2020). Gastrointestinal symptoms associated with COVID19: impact on the gut microbiome. Transl Res., 226:57-69.

Vuille-Dit-Bille, R. N.; Liechty, K. W.; Verrey, F and Guglielmetti, L. C. (2020). SARS-CoV-2 receptor ACE2 gene expression in small intestine correlates with age. Amino Acids, 52(6-7):1063-1065.

Wang, D.; Hu, B.; Hu, C.; Zhu, F.; Liu, X.; Zhang, J.; Wang, B.; Xiang, H.; Cheng, Z.; Xiong, Y.; Zhao, Y.; Li, Y.; Wang, X and Peng, Z. (2020). Clinical characteristics of 138 hospitalized patients with 2019 novel coronavirus-infected pneumonia in Wuhan, China. JAMA., 323(11):1061-1069.

Wang, J.; Zhao, S.; Liu, M.; Zhao, Z.; Xu, Y.; Wang, P.; Lin, M.; Xu, Y.; Huang, B.; Zuo, X.; Chen, Z.; Bai, F.; Cui, J.; Lew, A.M.; Zhao, J.; Zhang, Y.; Luo, H.B and Zhang, Y. (2020). ACE2 expression by colonic epithelial cells is associated with viral infection, immunity and energy metabolism. med Rxiv.

Wasimuddin Corman, V. M.; Ganzhorn, J.U; Rakotondranary, J.; Ratovonamana, Y.R.; Drosten, C and Sommer, S. (2019). Adenovirus infection is associated with altered gut microbial communities in a non-human primate. Sci. Rep., 9:e13410.

Wei, S.; Mortensen, M.S.; Stokholm, J.; Brejnrod, A.D.; Thorsen, J.; Rasmussen, M.A.; Trivedi, U.; Bisgaard, H and Sørensen, S.J. (2018). Short-and longterm impacts of azithromycin treatment on the gut microbiota in children: a double-blind, randomized, placebo-controlled trial. EBioMedicine, 38:265-272.

Weiss, G.A. and Hennet, T. (2017). Mechanisms and consequences of intestinal dysbiosis. Cell. Mol. Life Sci., 4:2959-2977.

Wu, H.J. and Wu, E. (2012). The role of gut microbiota in immune homeostasis and autoimmunity. Gut Microbes, 3(1):4-14

Wu, J.; Liu, J.; Zhao, X.; Liu, C.; Wang, W.; Wang, D.; Xu, W.; Zhang, C.; Yu, J.; Jiang, B and Cao, H. (2020). Clinical characteristics of imported cases of coronavirus disease 2019 (COVID-19) in Jiangsu Province: A multicenter descriptive study. Clin. Infect. Dis., 71(15):706-712.

Xiao, F.; Tang, M.; Zheng, X.; Liu, Y.; Li, X and Shan, H. (2020). Evidence for gastrointestinal infection of SARS-CoV-2. Gastroenterology, 158: $1831-1833$. 
Xu, K.; Cai, H.; Shen, Y.; Ni, Q.; Chen. Y.; Hu, S.; Li, J.; Wang, H.; Yu, L.; Huang, H and Qiu, Y. (2020). Management of coronavirus disease-19 (COVID19): the Zhejiang experience. J. Zhejiang Univ. Med. Sci., 49(1): 147-57.

Yang, T.; Chakraborty, S.; Saha, P.; Mell, B.; Cheng, X.; Yeo, J.Y.; Mei, X.; Zhou, G. Mandal, J.; Golonka, R.; Yeoh, B.S.; Putluri, V.; Piyarathna, D.W.B.; Putluri, N.; McCarthy, C.G.; Wenceslau, C.F.; Sreekumar, A.; Gewirtz, A.T.; VijayKumar, $M$ and Joe, B. (2004). Gnotobiotic rats reveal that gut microbiota regulates colonic mRNA of Ace2, the receptor for SARS-CoV-2 infectivity. Hypertension, 76(1):e1-e3.

Yazdanpanah, F.; Hamblin, M.R and Rezaei, N. (2020). The immune system and COVID-19: Friend or foe?. Life sciences, pp:117900.

Yeoh, Y.K.; Zuo, T.; Lui, G.C.Y.; Zhang, F.; Liu, Q.; Li,A.Y.; Chung, A.C.; Cheung, C.P.; Tso, E.Y.; Fung, K.S and Chan, V. (2021). Gut microbiota composition reflects disease severity and dysfunctional immune responses in patients with COVID-19. Gut., 70(4):698-706.

Yu, L. (2021). Restoring good health in elderly with diverse gut microbiome and food intake restriction to combat COVID-19. Ind J. Microbiol., 61(1): 104-107.

Zhang, C.; Wu, Z.; Li, J.W.; Zhao, H and Wang, G.Q. (2020a). Cytokine release syndrome in severe COVID-19: interleukin-6 receptor antagonist tocilizumab may be the key to reduce mortality. Int. J Antimicrob Agents., 55(5): 105954 .

Zhang, H.; Kang, Z.; Gong, H.; Xu, D.; Wang, J.; Li, Z.; Li, Z.; Cui, X.; Xiao, J.; Zhan, J.; Meng, T.; Zhou, W.; Liu, J and Xu, H. (2020b). Digestive system is a potential route of COVID-19: An analysis of single-cell coexpression pattern of key proteins in viral entry process. Gut., 69(6): 1010-1018.
Zhang, J.J.; Dong, X.; Cao, Y.Y.; Yuan, Y.D.; Yang, Y.B.; Yan, Y.Q.; Akdis, C.A and Gao, Y.D. (2020c). Clinical characteristics of 140 patients infected with SARS-CoV2 in Wuhan, China. Allergy, pp:1-12.

Zhao, Y.; Chen, F.; Wu, W.; Sun, M.; Bilotta, A.J.; Yao, S.; Xiao, Y.; Huang, X.; Eaves-Pyles, T.D.; Golovko, G and Fofanov, Y. (2018). GPR43 mediates microbiota metabolite SCFA regulation of antimicrobial peptide expression in intestinal epithelial cells via activation of mTOR and STAT3. Mucosal Immunol., 11(3):752-62.

Zhou, J.; Li, C.; Zhao, G.; Chu, H. Wang, D.; Yan, H.H.N.; Poon, V.K.M.; Wen, L.; Wong, B.H.Y.; Zhao, X.; Chiu, M.C.; Yang, D.; Wang, Y.; Au-Yeung, R.K.H.; Chan, I.H.Y.; Sun, S.; Chan, J.K.W.; To, K.K.W.; Memish, Z.A.; Corman, V.M.; Drosten, C.; Hung, I.F.N.; Zhou, Y.; Leung, S.Y and Yuen, K. Y. (2017). Human intestinal tract serves as an alternative infection route for Middle East respiratory syndrome coronavirus. Sci. Adv., 3:4966.

Zhou, P.; Yang, X.L.; Wang, X.G.; Hu, B.; Zhang, L.; Zhang, W.; Si, H.R.; Zhu, Y.; Li, B. and Huang, C.L.(2020). A pneumonia outbreak associated with a new coronavirus of probable bat origin. Nature, 579:270-273.

Zimmermann, M.; Zimmermann-Kogadeeva, M.; Wegmann, $\mathbf{R}$ and Goodman, A.L. (2019). Mapping human microbiome drug metabolism by gut bacteria and their genes. Nature, 570(7762):462-467.

Zuo, T.; Liu, Q.; Zhang, F.; Lui, G.C.; Tso, E.Y.; Yeoh, Y.K.; Chen, Z.; Boon, S.S.; Chan, F.K.; Chan, P.K and Ng, S.C. (2020a). Depicting SARS-CoV-2 faecal viral activity in association with gut microbiota composition in patients with COVID-19. Gut., 19:1-9.

Zuo, T.; Zhang, F.; Lui, G; Yeoh, Y. K.; Li,A.; Zhan, H.; Wan, Y.; Chung, A.; Cheung, C. P.; Chen, N.; Lai, C.; Chen, Z.; Tso, E.; Fung, K.; Chan, V.; Ling, L.; Joynt, G.; Hui, D.; Chan, F.; Chan, P. K.S and Ng, S. C. (2020b). Alterations in gut microbiota of patients with COVID-19 during time of hospitalization. Gastroenterology, 159(3):944-955. 